\title{
28 Research Square \\ 48-Year-Old Female MUTYH Carrier Presenting with Five Concurrent Primary Cancers.
}

\section{Aaron Arroyave}

The University of Tennessee Medical Center

\section{Laurentia Nodit}

The University of Tennessee Medical Center

Devin John Clegg ( $\nabla$ dclegg1@utmck.edu )

The University of Tennessee Medical Center https://orcid.org/0000-0002-5454-114X

\section{Andrew Russ}

The University of Tennessee Medical Center

\section{Research Article}

Keywords: Genetics, Oncology, Colon cancer, gynecology

Posted Date: March 10th, 2021

DOI: https://doi.org/10.21203/rs.3.rs-300783/v1

License: (c) (i) This work is licensed under a Creative Commons Attribution 4.0 International License. Read Full License 


\section{Abstract}

Background: MUTYH-associated polyposis (MAP) is a rare, and only recently described disorder resulting from mutations in genes encoding enzymes involved in DNA mismatch repair. These mutations result in an increased susceptibility to colonic adenomatosis as well as a variety of cancers. In current literature, studies have examined the frequencies of extracolonic manifestations of this disorder. However, these manifestations typically occur alone concurrently, or temporally separate from an already diagnosed colorectal cancer in individuals with a bi-allelic mutation.

Case Presentation: 48-year-old female with five distinct primary neoplasms presenting simultaneously in a patient mono-allelic for an MYH mutation, and only a single gastrointestinal polyp. Family history included colon, rectal, and lung cancer. Her past medical history included obesity and type 2 diabetes mellitus and a large ventral hernia was noted on examination. She initially presented with a severalmonth history of an enlarging pruritic labial mass found to represent a high grade squamous intraepithelial neoplasm for which a radical right partial vulvectomy was performed. Final pathology demonstrated a stage IB squamous cell carcinoma. At follow-up, the patient complained of fecal incontinence. To evaluate this, upper and lower endoscopies were performed which demonstrated a large rectal mass representing adenocarcinoma characterized by a low probability of microsatellite instability and an anal mass representing a second adenocarcinoma. An asymptomatic thyroid mass was discovered with pathology demonstrating papillary thyroid carcinoma. During her en bloc surgical resections of previously mentioned malignancies, a left ovarian serous implant of psammocarcinoma was identified. Scattered foci of low-grade serous carcinoma (psammomatous carcinoma) were found within the ventral hernia sac tissue.

Conclusions: The disparate and aggressive presentation of this case serves two purposes; to highlight the importance of a thorough workup in patients with oncologic presentations suspicious for genetic aberration, and to highlight how variable expressivity can lead to unpredictable presentations necessitating a broad differential diagnosis.

\section{Background:}

First described in 2002, MUTYH-associated polyposis (MAP) is an autosomal recessive familial colorectal cancer syndrome due to a mutation in the MYH gene [1]. Through its excision of misincorporated nucleotide residues caused by dihydro2'deoxyguanosine, a mutagenic product of oxidative DNA damage, adenine glycosylase, the enzyme encoded by this gene, functions in DNA mismatch repair [1,3-6]. When pathogenic MYH mutations occur, the phenotype expressed is typically characterized by extensive colonic adenomatosis, and an increased risk of colorectal and extracolonic malignancies. Colorectal cancer risk is increased between 23-to-28-fold in bi-allelic individuals. The presence of an increased risk of malignancy associated with mono-allelic mutations, particularly of the G396D variant seen in our patient, remains controversial [7]. The majority of current studies surrounding this topic sought to delineate the characteristics of this disease by identifying MYH mutations in populations of patients with polyposis, 
and without an identified familial syndrome. Concurrent extracolonic malignancies were frequently encountered, [2] however none as extensive as those seen in the case reported herein. Additionally, extensive colonic adenomatosis has historically been pathognomonic for Familial Adenomatous Polyposis. However, although only one gastrointestinal polyp was encountered in our case, studies have positively correlated the extent of polyposis with an increased likelihood of harboring an MYH mutation [8-10]. Therefore, in patients with multiple primary cancers, and patients with polyposis without an associated APC mutation, further genetic testing should be strongly considered. Due to the rarity of MAP, specific clinical screening and treatment guidelines have yet to be published. In light of this, the disparate and aggressive presentation of this case serves two educational purposes; to highlight the importance of a thorough workup in patients with oncologic presentations suspicious for genetic aberration, and to highlight how variable expressivity can lead to unpredictable presentations necessitating a broad differential diagnosis.

\section{Case Presentation:}

A 48-year-old female with family history of colon, rectal, and lung cancer (Figure 1.), and a past medical history of obesity and type 2 diabetes mellitus, presented in July 2018 with a several-month history of an enlarging pruritic labial mass found to represent a high grade squamous intraepithelial neoplasm for which a radical right partial vulvectomy was performed. Final pathology demonstrated a stage IB squamous cell carcinoma. Due to the presence of bilateral inguinal lymph node enlargement, a bilateral inguinal lymph node dissection was performed which demonstrated that all 15 lymph nodes removed were negative. At follow-up, the patient complained of fecal incontinence. To evaluate this, upper and lower endoscopies were performed which demonstrated a large rectal mass representing adenocarcinoma characterized by a low probability of microsatellite instability and an anal mass representing a second adenocarcinoma. No polyps were encountered.

In January 2019, colonoscopy revealed a large, circumferential, fungating, infiltrative, ulcerative, nonobstructing rectal mass. (Figure 2.) Biopsies of this mass demonstrated adenocarcinoma. (Figure 3.) In February 2019, further staging work-up included an elevated CEA level of $416.9 \mathrm{ng} / \mathrm{ml}$, a CT chest/abdomen/pelvis, and a pelvic MRI. In addition to demonstrating the rectal and anal neoplasms, (Figure 4.) the CT chest/abdomen/pelvis demonstrated a multinodular thyroid, a $1.5 \mathrm{~cm}$ right adrenal adenoma, a thin walled pulmonary nodule or cyst, bilateral iliac chain lymph node enlargement, a single enlarged portocaval lymph node, and a large ventral hernia. Staging pelvic MRI re-demonstrated the rectal lesion which was noted to be $15.6 \mathrm{~cm}$ in size, invading $50 \mathrm{~mm}$ in depth, extending from above the peritoneal reflection down to within $1 \mathrm{~cm}$ of the anal verge, possibly involving the pelvic floor musculature, posterior cervix, and vaginal cuff. Local lymph node involvement was radiographically suspected. (Figure 5.) Due to concern for local lymph node involvement and extracolonic extension, the patient was clinical stage IIIC (T4b, N1, Mx). The asymptomatic thyroid mass was evaluated via ultrasonography which demonstrated two TI-RADS 5 left-sided thyroid nodules, and a TI-RADS 4 right-sided thyroid nodule. (Figure 6.) Left lobe thyroid biopsies demonstrated papillary thyroid carcinoma. Due to the presence of multiple primaries, genetic testing was performed, and demonstrated heterozygosity for a p.G396D 
MUTYH mutation. The patient was negative for a multitude of other mutations including APC, MLH1, MSH2, MSH6, PMS2, BRCA 1\&2, MEN1, RET, PTEN, and TP53. Counselling was provided regarding associated risks, and early screening colonoscopy for her offspring was recommended.

The presentation of colorectal cancer alongside extracolonic malignancies raises suspicion for germline mutations and familial cancer syndromes. The presentation of our patient raised concern for Lynch Syndrome, Cowden disease, Familial Adenomatous Polyposis (FAP), and MYH-associated polyposis. The presentation of Lynch Syndrome is characterized by the development of colorectal cancers in addition to other extraintestinal cancer, including ovarian cancer. This, coupled with the relatively low number of intestinal polyps seen in Lynch Syndrome, consistent with our patient, further raised suspicion for this diagnosis. However, immunohistochemical stains for MLH1, MSH2, MSH6, and PMS2, which are used to screen for Lynch Syndrome, were negative. Additionally, though the patient did not demonstrate any germline mutations associated with Cowden syndrome, the diagnosis was considered due to her medical history of colon cancer, papillary thyroid cancer, and thyroid structural disease. However, the patient did not meet diagnostic criteria based on clinical history alone. In the current literature, MAP is most commonly diagnosed within patients suspected of having FAP, but without an APC mutation. While Attenuated FAP (AFAP) is characterized by a low number of polyps, absence of an APC mutation, again, excluded this diagnosis. The finding of a p.G396D MUTYH mutation indicated the most likely diagnosis to be MAP. This disorder is genetically defined by a homozygous mutation, and therefore, our patient, by current standards, should only be carrier and should not phenotypically express this typically Mendelian autosomal recessive disorder. However, the finding of this mutation, coupled with the development of characteristic extracolonic malignancies, calls into question the generalizability of this evolving diagnostic standard.

The patient underwent neoadjuvant chemoradiation prior to undergoing a total thyroidectomy with central lymph node dissection. Papillary carcinoma was found within both lobes, and 3 of 9 lymph nodes were positive for associated metastases. Radioactive iodine ablation was planned. A follow-up CT scan in May demonstrated a response by the rectal tumor to neoadjuvant chemoradiation, with a decrease in size of the rectal tumor from $8.7 \times 7.7 \times 10.7 \mathrm{~cm}$ to $6.6 \times 5.0 \times 6.9 \mathrm{~cm}$. (Figure 6.) In June, the patient underwent a posterior pelvic exoneration with a total abdominal hysterectomy and bilateral salpingooophorectomy. A sigmoid colectomy with en bloc radical vaginectomy and perineal proctectomy were performed. No gross metastatic foci were encountered, and the tumor was noted to be confined to the anorectum. The wound was closed primarily, a pelvic drain was placed, and a ventral hernia repair was performed. A single hyperplastic polyp was found in the colon. In addition to the two separate adenocarcinomas in the anus and rectum, a left ovarian serous implant of psammocarcinoma (Figure 7.) was identified. Scattered foci of low grade serous carcinoma (psammomatous carcinoma) were found within the ventral hernia sac tissue. All twenty-two lymph nodes were negative for malignancy. The patient was discharged on postoperative day 3 after an uncomplicated hospital stay.

The patient was discharged to a skilled nursing facility for continued wound care. Ongoing plans include adjuvant therapy for her anorectal cancer, as well as further therapy to treat her low grade peritoneal 
primary serous cancer. This patient's disease was investigated by both our genetics and dermatology departments thoroughly, and was presented at a multidisciplinary conference to discuss the possibility of Cowden Syndrome. They further recommended obtaining genetic testing for her children which is being considered.

\section{Discussions And Conclusions:}

The case presented contains several peculiarities which merit discussion and defy the currently tenuous conclusions surrounding this rare disease. The two most common MYH mutations, of the greater than 60 mutations currently identified, include Y179C and G396D mutations [11]. In the current literature, the risk of colorectal cancer due to a bi-allelic MYH mutation is increased between 23-to-28-fold, depending on the specific mutations. However, the presence of an increased risk of malignancy associated with monoallelic mutations, particularly of the less virulent G396D variant seen in our patient, has yet to be established [7]. While G396D mutations, and the types of extracolonic malignancies which developed in our patient, are characteristic of MAP, the heterozygosity of her mutation might indicate an additional unidentified genetic aberration resulting in an increased susceptibility to malignant degeneration. In some aspects, the clinical presentation of our patient conforms strongly with the diagnosis of MAP, especially with regards to the types of extracolonic malignancies developed. Ovarian, thyroid, and skin cancer have all been found in individuals with MAP-associated colorectal cancer [2, 12-14]. The risks of developing extracolonic malignancies have not been respectively identified. However, Vogt et al. calculated a 38\% overall lifetime risk of developing extracolonic malignancy in patients with MAP. The relative absence of polyps, as seen in our patient, is uncharacteristic of MAP. A study by Wang et al. found that the presence of a bi-allelic germline MYH mutation correlated with the presence of $\geq 20$ adenomatous polyps [9]. In one study of 152 patients with polyposis, among patients with adenomas ranging in number between 3 and 100, biallelic MYH mutations were found only in individuals with greater than 15 polyps [10]. Additionally, of the 25 MAP patients within the study by Sampson et al, eleven patients had between ten and 100 adenomas, nine had greater than 100 , and five had adenomas too numerous to count [8]. This unique presentation of an MUTYH mutation raises suspicion for concurrent and undetected genetic abnormalities. However, because MAP remains rare, and consequentially, studies regarding its prevalence and natural history remain relatively inconclusive, it is possible that this case only highlights how much left there is to learn about this disease. Formal screening and management guidelines specific for this disease have yet to be developed. The importance of understanding MAP, and thus diagnosing it and treating it appropriately, lies in its clinical similarity to other well characterized familial cancer syndromes. Its autosomal recessive nature directly contrasts the autosomal dominant nature of FAP; the diagnosis most commonly ruled out prior to its diagnostic consideration. This difference has implications regarding which family members to screen, and when to screen them. Additionally, its clinical presentation is similar to, but distinct from, other familial cancer syndromes. Therefore, a distinct work-up is likely necessary, however one has yet to be independently established. Prospective studies on MAP have not yet been conducted. They would doubtlessly be invaluable to its accurate characterization and elucidation of its proper diagnosis and management. 


\section{Abbreviations}

MAP: MUTYH-associated polyposis

CEA: Carcinoembryonic antigen

TI-RADS: Thyroid Imaging Reporting \& Data System

FAP: Familial Adenomatous Polyposis

AFAP: Attenuated Familial Adenomatous Polyposis

\section{Declarations}

\section{Ethics approval and consent to participate:}

Patient agreed to participate in the publication of this case report, provided consent, and hopes that it serves to increase understanding of her disease process. Ethics approval not required.

\section{Consent for publication:}

Consent has been obtained by the patient for reporting of this case.

\section{Availability of data and materials:}

Data sharing is not applicable to this article as no datasets were generated or analysed during the current study.

\section{Competing interests:}

The authors declare that they have no competing interests.

\section{Funding}

The authors have no funding sources to declare.

\section{Authors' contributions:}

AA- Assisted with patient care, development of manuscript and figures, interpretation of genetic analysis and literature review. 
LN- Assisted with patient care, performed histological examinations, creation of figures, development of manuscript.

DC- Development of manuscript, figures, correspondence, manuscript submission.

AR- Primary patient care, surgical intervention, development of manuscript.

Acknowledgements:

We would like to acknowledge the contributions of the Department of Surgical Oncology, Department of Obstetrics and Gynecology, Department of Pathology, University Genetics and all others that assisted in patient care.

\section{References}

1. Jones S, Emmerson P, Maynard J, Best JM, Jordan S, Williams GT, et al. Biallelic germline mutations in $\mathrm{MYH}$ predispose to multiple colorectal adenoma and somatic $\mathrm{G}: \mathrm{C}->\mathrm{T}$ :A mutations. Hum Mol Genet. 2002;11(23):2961-7.

2. Vogt S, Jones N, Christian D, Engel C, Nielsen M, Kaufmann A, et al. Expanded extracolonic tumor spectrum in MUTYH-associated polyposis. Gastroenterology. 2009;137(6):1976-85.e1-10.

3. LindahI T. Instability and decay of the primary structure of DNA. Nature. 1993;362(6422):709-15.

4. Michaels ML, Miller JH. The GO system protects organisms from the mutagenic effect of the spontaneous lesion 8-hydroxyguanine (7,8-dihydro-8-oxoguanine). J Bacteriol. 1992;174(20):63215.

5. Slupska MM, Baikalov C, Luther WM, Chiang JH, Wei YF, Miller JH. Cloning and sequencing a human homolog (hMYH) of the Escherichia coli mutY gene whose function is required for the repair of oxidative DNA damage. J Bacteriol. 1996;178(13):3885-92.

6. Parker A, Gu Y, Mahoney W, Lee SH, Singh KK, Lu AL. Human homolog of the MutY repair protein ( $\mathrm{hMYH}$ ) physically interacts with proteins involved in long patch DNA base excision repair. J Biol Chem. 2001;276(8):5547-55.

7. Theodoratou E, Campbell H, Tenesa A, Houlston R, Webb E, Lubbe S, et al. A large-scale metaanalysis to refine colorectal cancer risk estimates associated with MUTYH variants. $\mathrm{Br} J$ Cancer. 2010;103(12):1875-84.

8. Sampson JR, Dolwani S, Jones S, Eccles D, Ellis A, Evans DG, et al. Autosomal recessive colorectal adenomatous polyposis due to inherited mutations of MYH. Lancet. 2003;362(9377):39-41.

9. Wang L, Baudhuin LM, Boardman LA, Steenblock KJ, Petersen GM, Halling KC, et al. MYH mutations in patients with attenuated and classic polyposis and with young-onset colorectal cancer without polyps. Gastroenterology. 2004;127(1):9-16.

10. Sieber OM, Lipton L, Crabtree M, Heinimann K, Fidalgo P, Phillips RK, et al. Multiple colorectal adenomas, classic adenomatous polyposis, and germ-line mutations in MYH. N Engl J Med. 
2003;348(9):791-9.

11. Sampson JR, Jones N. MUTYH-associated polyposis. Best Pract Res Clin Gastroenterol. 2009;23(2):209-18.

12. Aretz S, Uhlhaas S, Goergens H, Siberg K, Vogel M, Pagenstecher C, et al. MUTYH-associated polyposis: 70 of 71 patients with biallelic mutations present with an attenuated or atypical phenotype. Int J Cancer. 2006;119(4):807-14.

13. Ponti G, Ponz de Leon M, Maffei S, Pedroni M, Losi L, Di Gregorio C, et al. Attenuated familial adenomatous polyposis and Muir-Torre syndrome linked to compound biallelic constitutional MYH gene mutations. Clin Genet. 2005;68(5):442-7.

14. Pervaiz MA, Eppolito A, Schmidt K. Papillary thyroid cancer in a patient with MUTYH-associated polyposis (MAP). Fam Cancer. 2010;9(4):595-7.

\section{Figures}




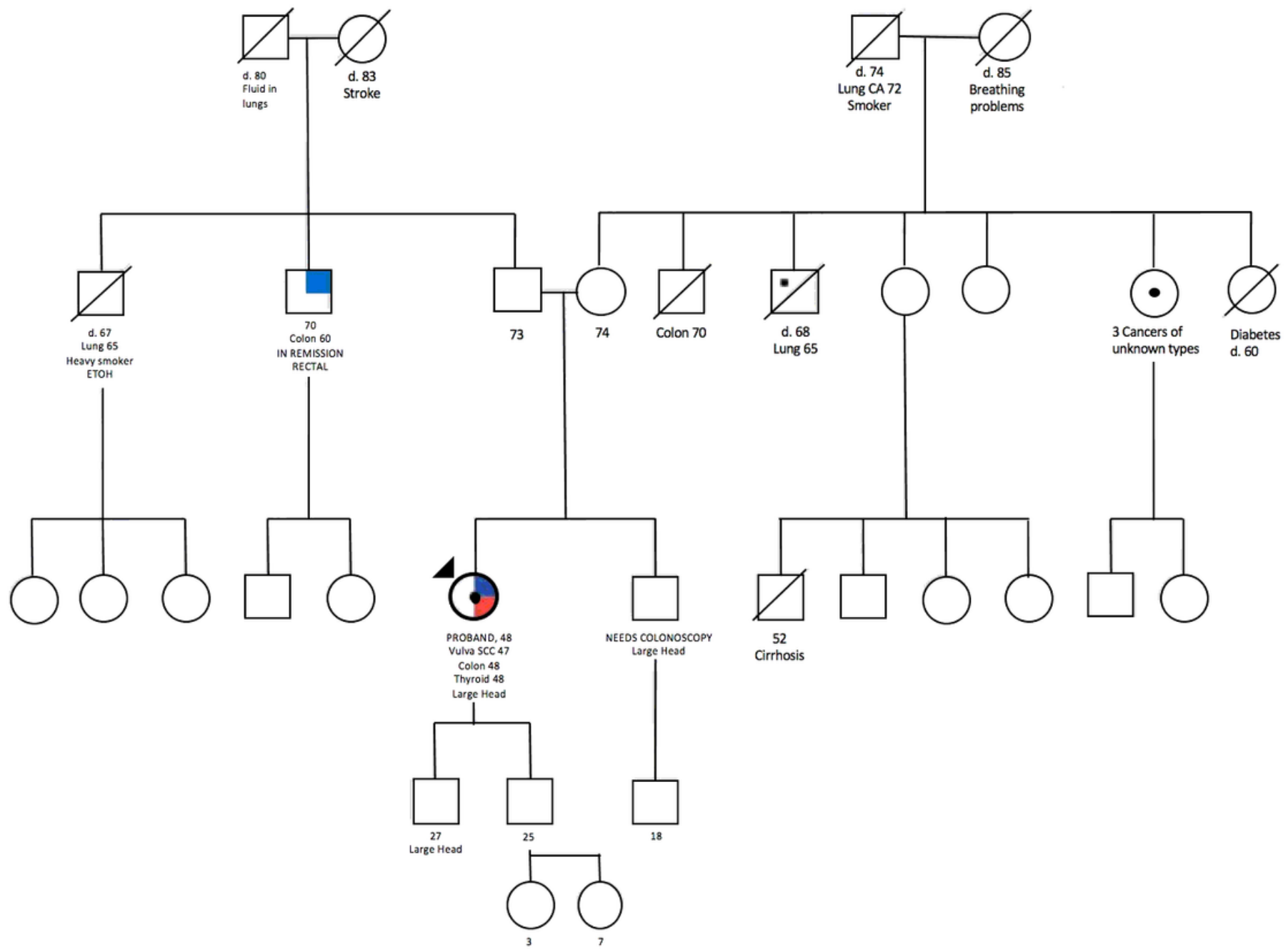

Figure 1

\section{Pedigree.}
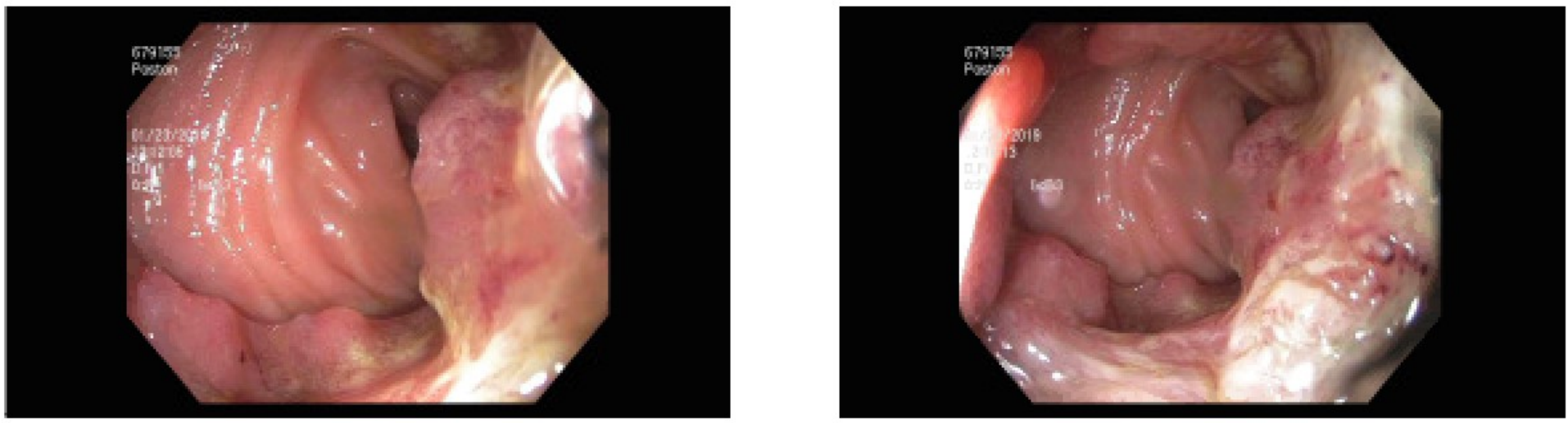

Figure 2 
Anorectal mass on colonoscopy characterized as fungating, infiltrative, polypoid, sessile, ulcerative, nonobstructing, and circumferential.



Figure 3

Microscopic photograph showing adenocarcinoma. Hematoxylin- Eosin Stain, 200x magnification. 

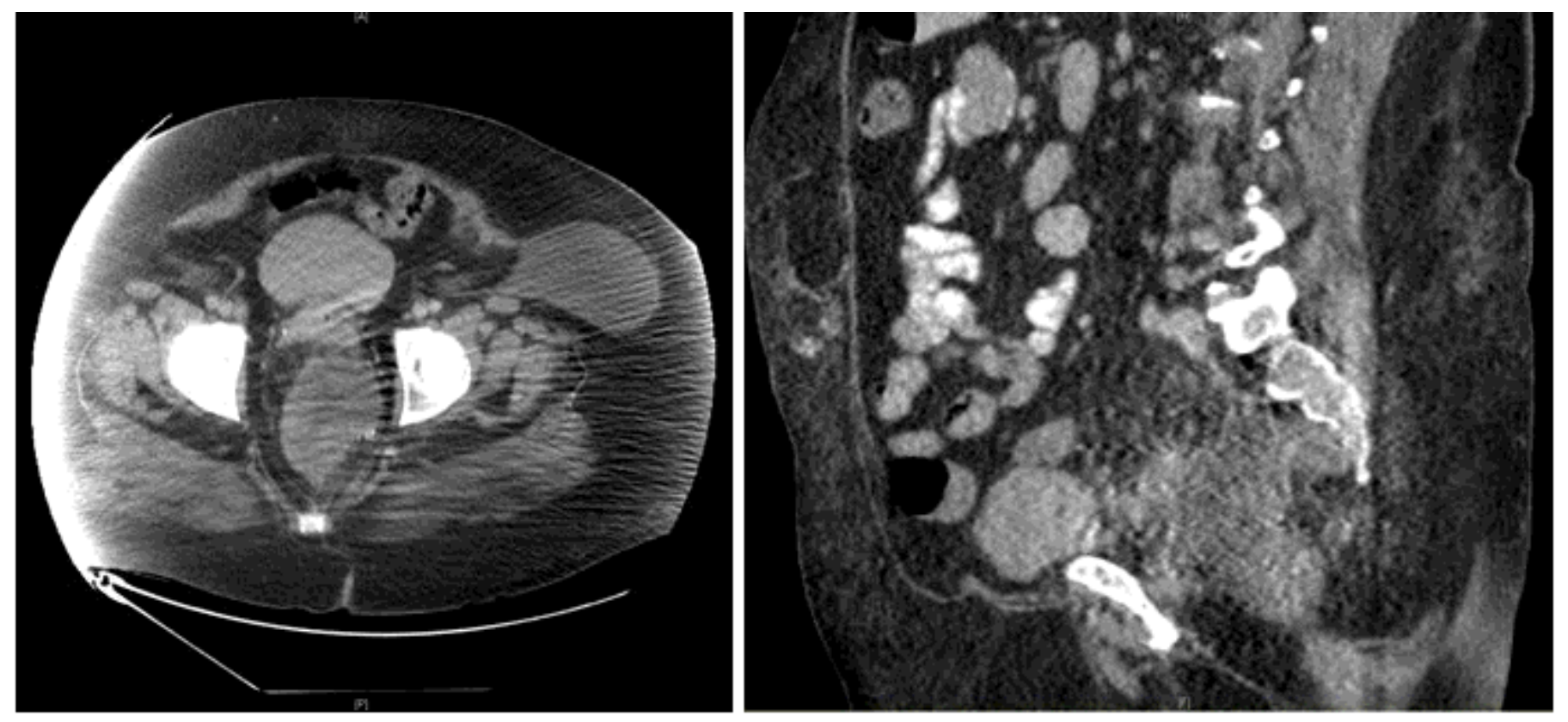

Figure 4

CT chest/abdomen/pelvis: Anorectal mass axial and sagittal view 


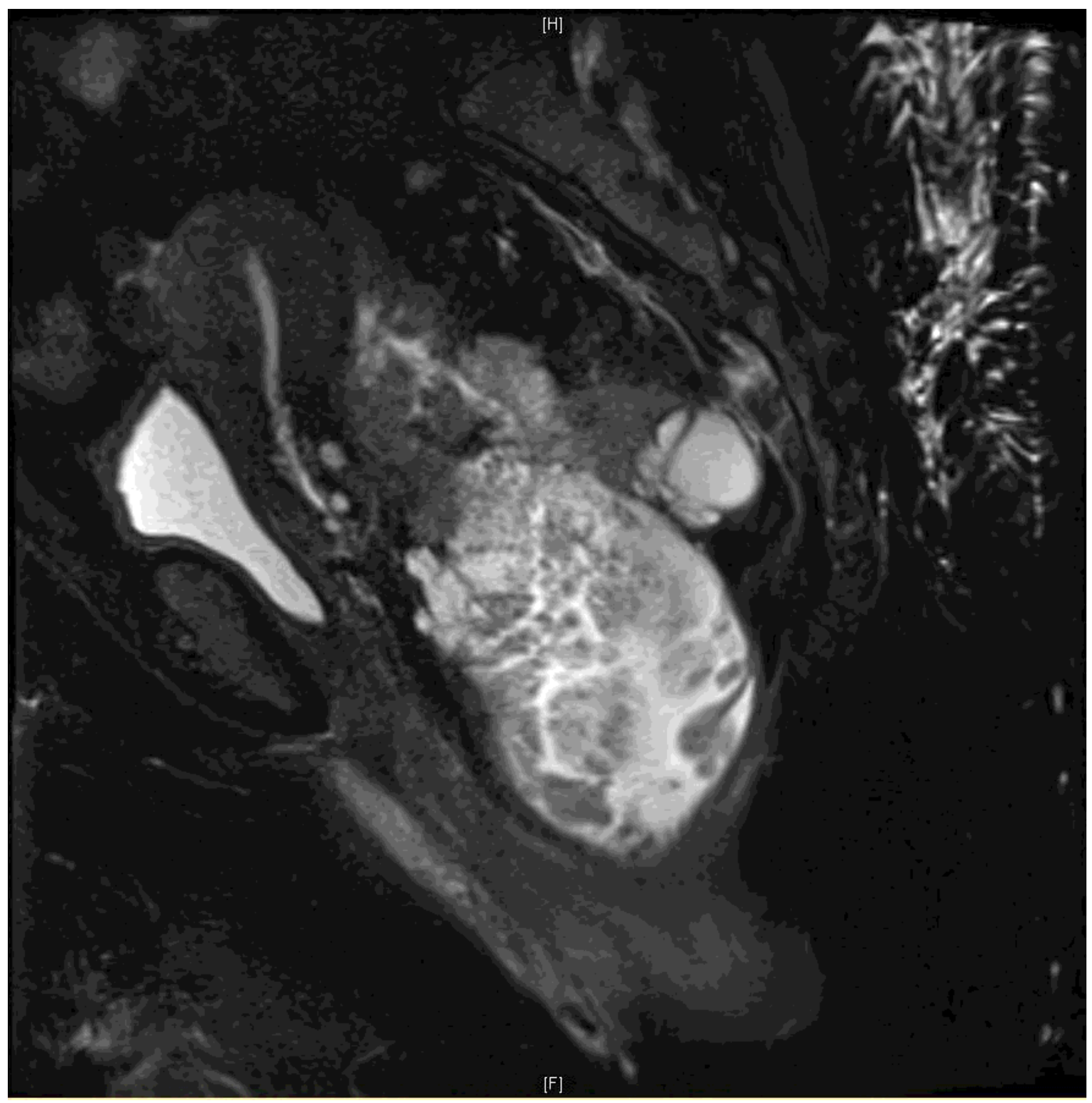

\section{Figure 5}

MRI pelvis demonstrated a circumferential, fungating, mucinous, $15.6 \mathrm{~cm}$ mass within $1 \mathrm{~cm}$ of the anal verge, extending from above the peritoneal reflection down to the lower rectum and upper anal canal. The tumor involved the puborectalis, and internal and external anal sphincters, and was noted to have a 50$\mathrm{mm}$ depth of invasion. Due to abutment of the posterior cervix, vaginal cuff, and bilateral levator muscles, the tumor was believed to invade adjacent organs and was staged as a T4b tumor. The MRI also 
suspicious for local lymph node involvement, therefore patient was deemed to be clinical stage IIIC (T4b, N1, Mx).

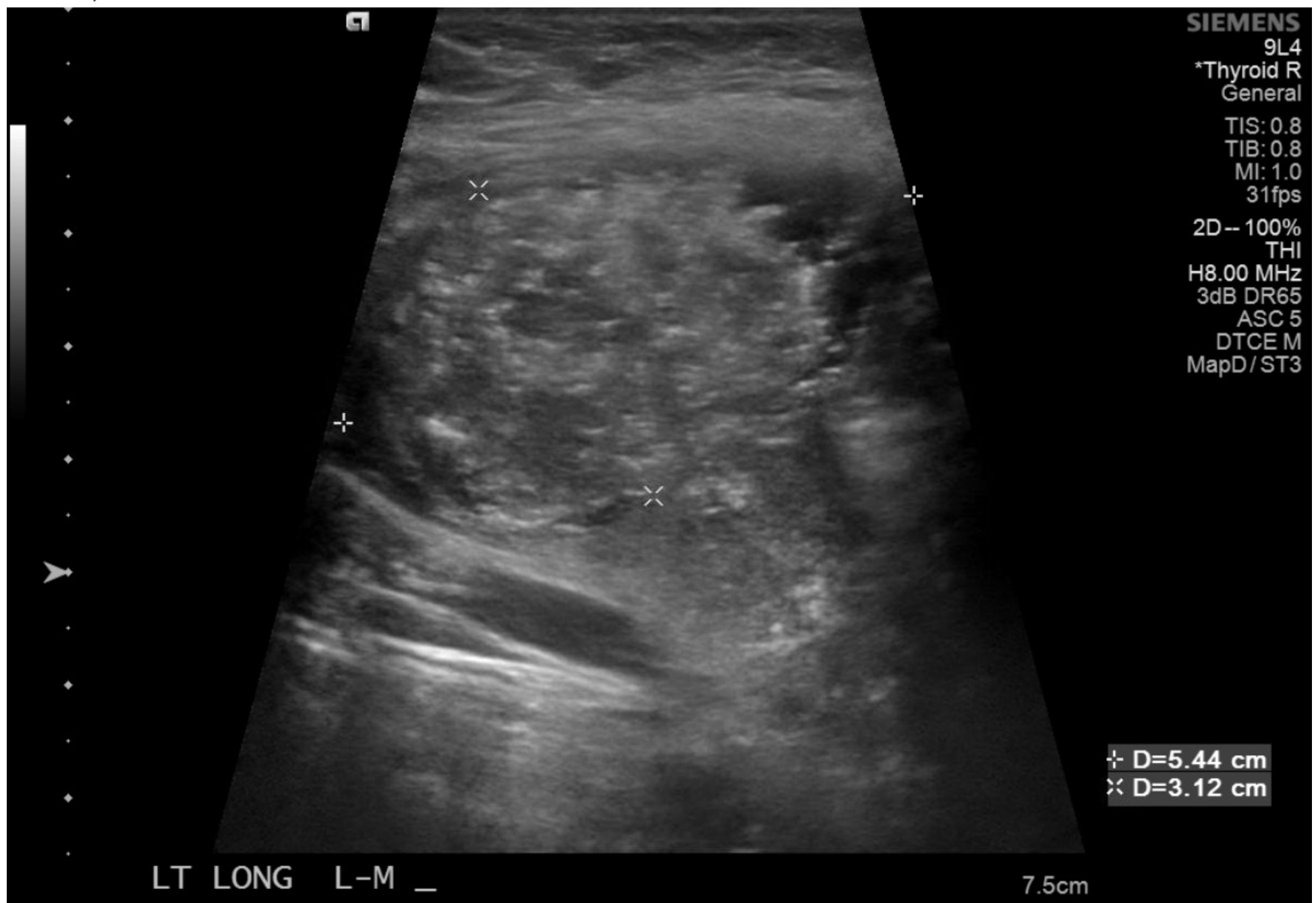

\section{Figure 6}

Thyroid ultrasound demonstrating multiple bilateral nodules concerning for malignancy. Pictured below is a TI-RADS 5, left-sided thyroid nodule, approximately $5.4 \mathrm{~cm}$ in greatest dimension. 


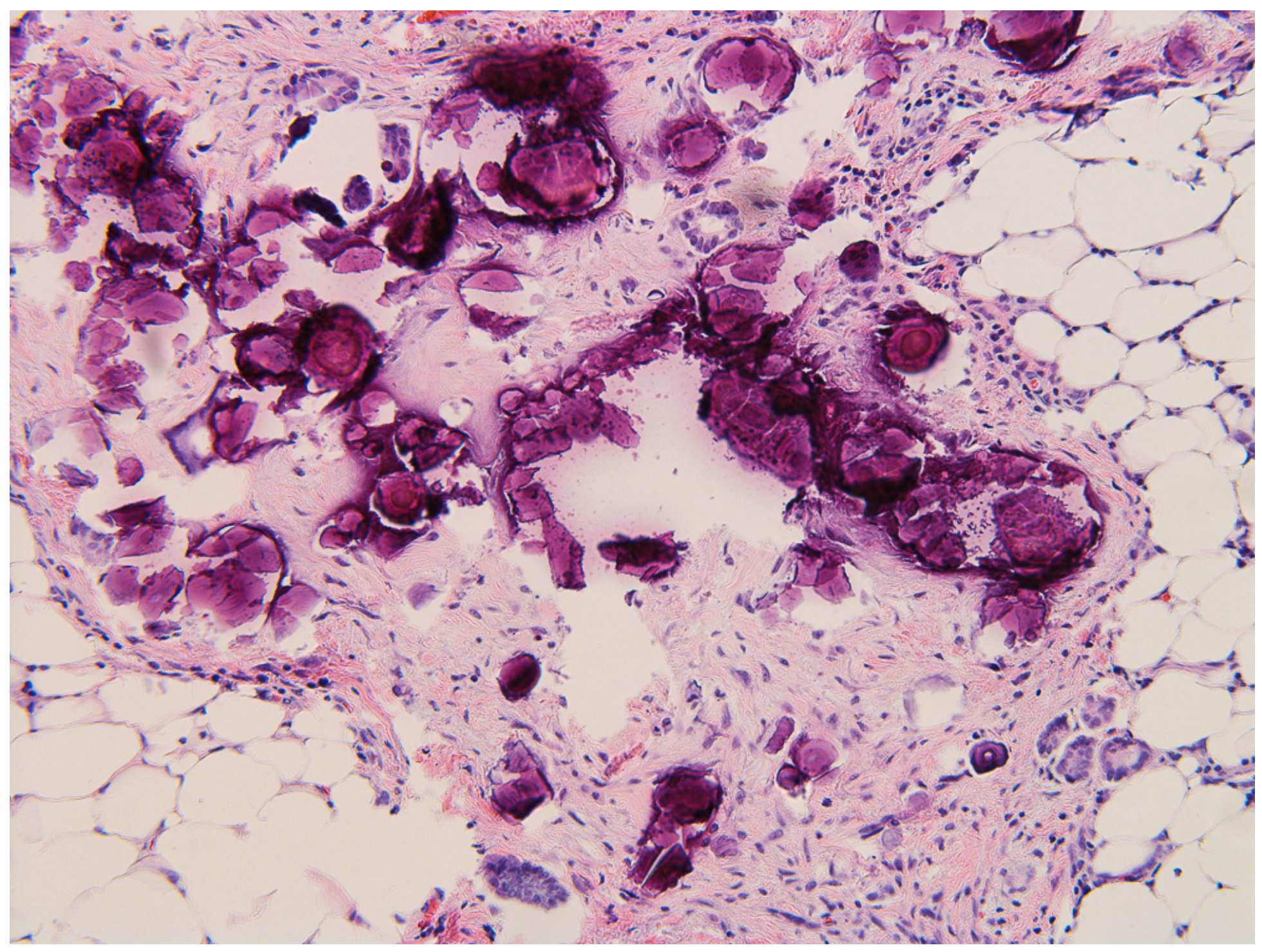

Figure 7

Serous peritoneal psammocarcinoma histology stained positive for MOC 31, Ber-EP4, WT-1, PAX8, and Estrogen receptor. Stained negative for Calretinin, D2-40, p53, and TTF-1. 